\title{
Anisotropic hole spins in single and coupled self-assembled quantum dots
}

\author{
Weidong Sheng* \\ Department of Physics, Fudan University, Shanghai 200433, China and Department of Physics, University of Hong Kong, \\ Hong Kong, China \\ Jian Wang \\ Department of Physics, University of Hong Kong, Hong Kong, China
}

(Received 25 April 2010; published 30 August 2010)

\begin{abstract}
Anisotropy of hole spins in single and coupled self-assembled InAs/GaAs quantum dots is studied theoretically by means of an effective bond-orbital method. Compared with isotropic electron spins within the growth plane, it is found that the hole spins, although with much smaller positive $g$ factors, can be highly anisotropic in single quantum dots. It is shown that the in-plane anisotropy of the hole spins can even reverse its sign as the height of the dots varies. In vertically coupled quantum dots, the in-plane hole $g$ factors become comparable to the electrons, almost one order of magnitude larger than those in the single dots. Our result agrees qualitatively with the recent experiment [S. A. Crooker et al., Phys. Rev. Lett. 104, 036601 (2010)].
\end{abstract}

DOI: 10.1103/PhysRevB.82.073308

PACS number(s): 71.18.+y, 78.67.Hc, 73.21.La

Electron spin, as one of the fundamental degrees of freedom, has attracted much interest due to its potential application in spintronic devices. ${ }^{1}$ Semiconductor quantum dots have been proposed as promising candidates to realize quantum bits based on electron spin. ${ }^{2}$ In most of semiconductors such as InAs and GaAs, electron spin is usually isotropic and hence its $g$ factor tensor reduces to a scalar. However, as the spin of hole is strongly coupled to its orbital motion of localized spin-orbitals, its $g$ factor is identified by the different angular momenta and can be highly anisotropic. In self-assembled InAs/GaAs quantum dots, hole states are generally dominated by the heavy-hole components and mixed with minor light-hole and split-hole parts due to the quantum confinement effect and inhomogeneous strain distribution. ${ }^{3}$ Each of the constituent component has distinct $g$ factor with different anisotropy, which has been shown to play an important role in determining the properties of electron spins. ${ }^{4}$

In the presence of magnetic field applied within the growth plane of self-assembled InAs/GaAs quantum dots, the hole $g$ factors are known to be much smaller than those of the electrons, e.g., it is found that, in InAs/GaAs quantum dots, $\left|g_{h}\right|=0.15$ (Ref. 5) and $\left|g_{h}\right|=0.24$ (Ref. 6) compared with the corresponding $\left|g_{e}\right|=0.54$ and $\left|g_{e}\right|=0.47$. Unlike an almost isotropic electron spin within the growth plane, ${ }^{5}$ it is found that a hole spin exhibits remarkable in-plane anisotropy in a recent experiment. ${ }^{7}$ More surprisingly, the anisotropy of the hole $g$ factors can even change its sign with the emission energy.

In this work, we will study the in-plane anisotropy of the hole effective $g$-factors in self-assembled quantum dots. Applied magnetic field takes direction parallel to the growth plane of the quantum dots, commonly known as Voigt configuration. Self-assembled InAs/GaAs quantum dots are assumed to take lens-shaped or truncated pyramidal geometry and have $2 \mathrm{ML}$ of wetting layer. Following the calculation of strain distribution by the valence-force-field method, we adopt an effective bond-orbital approach to calculate $g$ factors of the electronic states in quantum dots. The Hamiltonian reads

$$
\begin{gathered}
H\left(\mathbf{R}_{s \sigma}, \mathbf{R}_{s \sigma}^{\prime}\right)=E_{s s}^{000} \delta_{\mathbf{R}, \mathbf{R}^{\prime}}+E_{s s}^{110} \delta_{\Delta \mathbf{R}, \tau}+E_{s s}^{200} \delta_{\Delta \mathbf{R}, \lambda}, \\
H\left(\mathbf{R}_{p \sigma}, \mathbf{R}_{p \sigma}^{\prime}\right)= \\
E_{x x}^{000} \delta_{\mathbf{R}, \mathbf{R}^{\prime}}+\delta_{\Delta \mathbf{R}, \tau}\left[E_{x x}^{110} \tau_{p}^{2}+E_{x x}^{011}\left(1-\tau_{p}^{2}\right)\right] \\
+\delta_{\Delta \mathbf{R}, \lambda}\left[E_{x x}^{200} \sigma_{p}^{2}+E_{x x}^{002}\left(1-\sigma_{p}^{2}\right)\right] \\
H\left(\mathbf{R}_{s \sigma}, \mathbf{R}_{p \sigma}^{\prime}\right)=E_{s x}^{110} \tau_{p} \delta_{\Delta \mathbf{R}, \tau}, \\
H\left(\mathbf{R}_{p \sigma}, \mathbf{R}_{p^{\prime} \sigma}^{\prime}\right)=E_{x y}^{110} \tau_{p} \tau_{p^{\prime}} \delta_{\Delta \mathbf{R}, \tau},
\end{gathered}
$$

where $\delta_{\mathbf{R}, \mathbf{R}^{\prime}}$ is the Kronecker delta symbol, $\Delta \mathbf{R}=\mathbf{R}-\mathbf{R}^{\prime}$, and $\tau_{p}$ and $\lambda_{p}$ are the positions of the nearest and next-nearest neighbors, respectively. The tight-binding parameters for GaAs and InAs can be found in one of our previous work. ${ }^{4} \mathrm{~A}$ geometric averaging is applied to the matrix elements across two neighboring GaAs and InAs unit cells. Magnetic field is incorporated into the tight-binding Hamiltonian by introducing Peierls phase factor. ${ }^{8}$ Off-diagonal matrix elements are transformed as

$$
H\left(\mathbf{R}, \mathbf{R}^{\prime}\right) \rightarrow e^{-i(e / \hbar) \int_{\mathbf{R}}^{\mathbf{R}^{\prime}} \mathbf{A}(\mathbf{r}) d \mathbf{r}} \cdot H\left(\mathbf{R}, \mathbf{R}^{\prime}\right),
$$

where $\mathbf{A}(\mathbf{r})$ is the vector potential. Zeeman terms are added to the diagonal matrix elements,

$$
H\left(\mathbf{R}_{\uparrow(\downarrow)}, \mathbf{R}_{\uparrow(\downarrow)}\right) \rightarrow H\left(\mathbf{R}_{\uparrow(\downarrow)}, \mathbf{R}_{\uparrow(\downarrow)}\right) \pm \frac{1}{2} g_{0} \mu_{B} B,
$$

where $g_{0}$ is the $g$ factor of a bare electron. The matrix elements for spin-orbit interaction are given by $H\left(\mathbf{R}_{x \uparrow}, \mathbf{R}_{y \uparrow}\right)=-i \Delta / 3, H\left(\mathbf{R}_{x \downarrow}, \mathbf{R}_{y \downarrow}\right)=i \Delta / 3, H\left(\mathbf{R}_{x \uparrow}, \mathbf{R}_{z \downarrow}\right)=\Delta / 3$, $H\left(\mathbf{R}_{y \uparrow}, \mathbf{R}_{z \downarrow}\right)=-i \Delta / 3$, and their conjugate terms, with $\Delta$ the spin-orbit splitting in valence bands.

The first model system we choose is a lens-shaped InAs/ GaAs quantum dot as shown in the inset of Fig. 1. Please be noted that the dots studied in the experiment ${ }^{7}$ are annealed after the growth. The fact that other similarly annealed dots exhibit Fock-Darwin diagram ${ }^{9,10}$ in their emission spectra implies that the dots are most likely lens-shaped because only lens-shaped dots may have almost equally spaced en- 


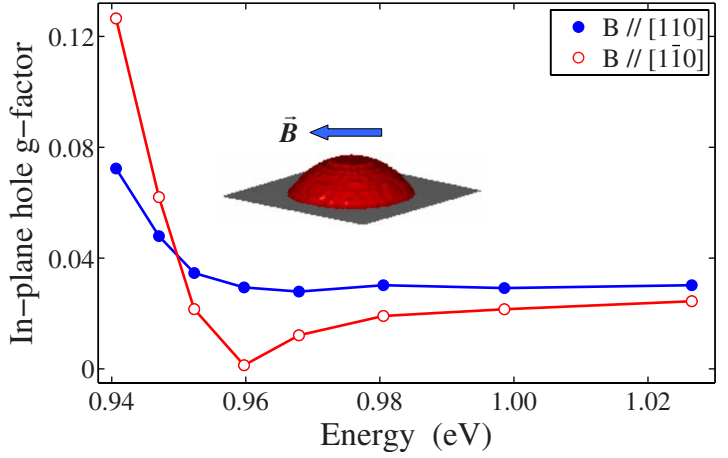

FIG. 1. (Color online) Hole $g$-factor in an InAs/GaAs selfassembled quantum dot calculated as a function of the emission energy for the magnetic field directed along the [110] (solid dots) and $[1 \overline{1} 0]$ (open dots) directions, respectively. The lens-shaped dot has a diameter of $19.8 \mathrm{~nm}$ and height varying between $2.8 \mathrm{~nm}$ and $6.8 \mathrm{~nm}$. Cubic spline fits are shown in solid lines as the guide of the eye.

ergy levels. ${ }^{11}$ The base diameter is fixed at $19.8 \mathrm{~nm}$ and height varies between $2.8 \mathrm{~nm}$ and $6.8 \mathrm{~nm}$. The corresponding emission energy lies between about $0.94 \mathrm{eV}$ and $1.03 \mathrm{eV}$. Figure 1 plots the hole $g$ factor calculated as a function of the emission energy in Voigt configuration. The modulus of the hole $g$ factor is determined from the spin splitting of the hole states at $B=1$ T. The sign of the hole $g$ factor can be resolved from that of the electron. The electron $g$ factor in InAs/GaAs quantum dots is shown in our previous study ${ }^{3}$ to always carry a negative sign in Faraday configuration, which agrees with the experiment. ${ }^{5}$ Our later study reveals that the electron $g$ factor satisfies a perfect fitting by $|g(\theta)|$ $=\sqrt{\left(g_{F} \cos \theta\right)^{2}+\left(g_{V} \sin \theta\right)^{2}}$ between Faraday and Voigt configurations. ${ }^{12}$ As $g(\theta)$ has to be a continuous function, it can be therefore deduced that the electron $g$ factor should also be negative in Voigt configuration. For the system as shown in Fig. 1, we find that the modulus of exciton $g$ factor is determined by $\left|g_{e}\right|-\left|g_{h}\right|$ instead of $\left|g_{e}\right|+\left|g_{h}\right|$, hence the hole $g$ factor mush have an opposite sign to that of the electron, which is positive. ${ }^{5}$

The hole $g$-factors are calculated at the selected angles and are fit to $g^{2}=g_{x}^{2} \cos ^{2}\left(\alpha-\alpha_{0}\right)+g_{y}^{2} \sin ^{2}\left(\alpha-\alpha_{0}\right)$ where we find $\alpha_{0}$ is very close to $45.0^{\circ}$, i.e., the [110] direction, for all the dots. Therefore, we plot the results only for the magnetic field applied along the [110] and [1 $\overline{10}]$ directions. It is surprising to find that the major axis of the in-plane hole $g$ factor is not always the crystal [110] axis. For the thicker dots with lower emission energy, the [110] direction is seen to become the major axis, which agrees with the experiment. $^{7}$

The anisotropy of in-plane hole $g$ factors is defined by

$$
p=\frac{g_{110}-g_{1 \overline{10}}}{g_{110}+g_{1 \overline{10}}} .
$$

We find that it reverses its sign with the increasing emission energy as seen in Fig. 2. Around $0.96 \mathrm{eV}, g_{[110]}$ is very small (0.0013) and hence the anisotropy is seen to reach almost

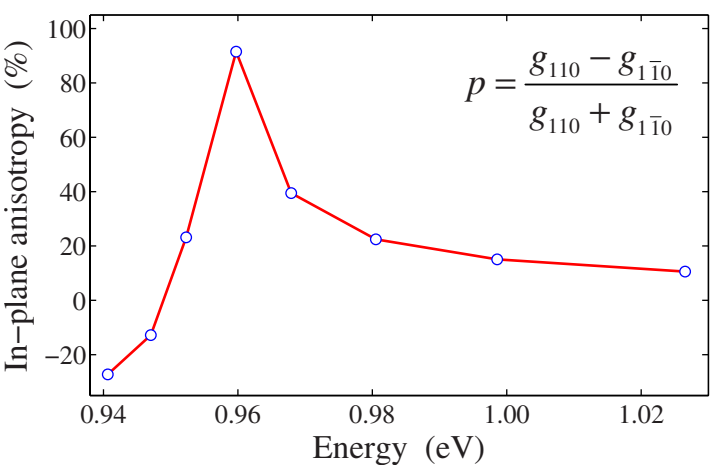

FIG. 2. (Color online) Anisotropy of in-plane hole $g$ factors calculated as a function of the emission energy for the same dots as Fig. 1. Cubic spline fits are shown in solid lines as the guide of the eye.

$100 \%$. Except for the extreme, the in-plane anisotropy lies between $-20 \%$ and $40 \%$.

For a dot with small aspect ratio between the vertical and lateral dimensions, we notice that the difference in the energy spectrum between lens-shaped and truncated pyramidal is not significant. Therefore, the calculation has also been performed for truncated pyramidal InAs dots and moderately intermixed $\mathrm{In}_{0.6} \mathrm{Ga}_{0.4} \mathrm{As}$ lens-shaped dot. The former has a base length of $19.8 \mathrm{~nm}$ and height of $5.1 \mathrm{~nm}$, and the latter has a same diameter as the previous lens-shaped systems. Here, randomness is introduced to only the level of unit cell. Figure 3 plots the angular dependence of the hole $g$ factors

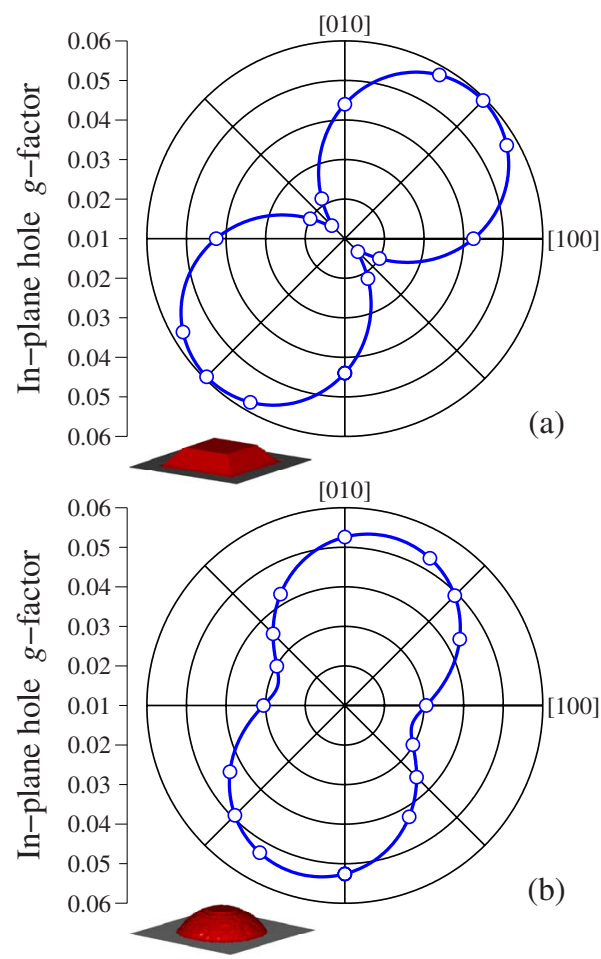

FIG. 3. (Color online) In-plane angular dependence of the calculated hole $g$ factor (circles) and the fits to data (solid lines) for a truncated pyramidal InAs/GaAs dot with a base length of $19.8 \mathrm{~nm}$ (a) and an intermixed lens-shaped $\mathrm{In}_{0.6} \mathrm{Ga}_{0.4} \mathrm{As} / \mathrm{GaAs}$ dot with a diameter of $19.8 \mathrm{~nm}$ (b). The height of the two dots is $5.1 \mathrm{~nm}$. 


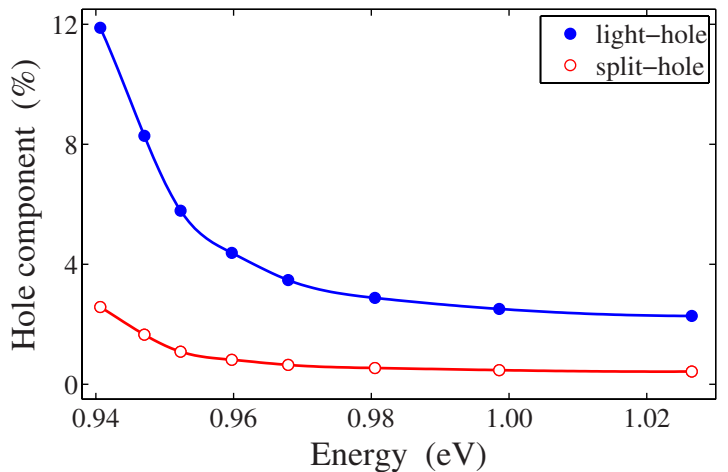

FIG. 4. (Color online) The proportion of the light- and split-hole components in the ground state of holes calculated as a function of the emission energy for the same dots as Fig. 1.

calculated for these two model systems. It is found that the hole $g$ factor in the pure truncated pyramidal InAs dot exhibits very similar pattern as that in the lens-shaped model. The major axis of the in-plane hole $g$ factor is seen to be perfectly lying along the crystal [110] axis. However, we find that the lens-shaped model agrees better with the experiment in terms of energetic dependence of the hole $g$ factor. As for the intermixed model, due to the randomly distributed Gallium content inside the dot island, the major axis of the inplane hole $g$ factor is found to be along neither the [110] nor $[1 \overline{1} 0]$ directions. The result for the intermixed samples suggests that the in-plane anisotropy of the hole $g$ factors has strong relation with the spatial distribution of the hole wave functions. This conclusion, however, is opposite to that found for the in-plane electron $g$ factors which are always isotropic even in the presence of intermixing effect.

By decomposing a hole state $\psi$ into $\psi_{s}|s\rangle+\psi_{h h}|h h\rangle+\psi_{\ell h}|\ell h\rangle+\psi_{s h}|s h\rangle$ and introducing an empirical parameter $\kappa,{ }^{12}$ the in-plane hole $g$ factors can be expressed as the sum of the contributions from each component $g=-4 \kappa \cdot \rho_{\ell h}-2 \kappa \cdot \rho_{s h}$, where $\rho_{k}=\left|\left\langle\psi_{k} \mid \psi_{k}\right\rangle\right|^{2}$. Here, we ignore the contribution from the $s$ component which is very small and that from the heavy-hole component whose spin is frozen along the growth direction. ${ }^{12}$ The proportion of the lightand split-hole components in the ground state of holes is plotted in Fig. 4 as a function of emission energy for the same dot as shown in Fig. 1. Considering that the empirical parameter $\kappa$ is positive, ${ }^{4}$ it is therefore seen that the hole $g$ factor in Voigt configuration shall be isotropic and always be negative.

This nominal contradiction between the phenomenological model and numerical calculation can be resolved by taking into account the nonzero envelope orbital momenta (NEOM) carried by the hole states. ${ }^{3}$ As has been shown, the positive contribution from NEOM would give rise to an overall positive hole $g$ factor. Furthermore, the positive contribution from NEOM depends on the direction along which the magnetic field is applied because the probability of the hole wave function is anisotropic within the growth plane especially for those thick dots. ${ }^{13}$ It explains why the in-plane anisotropy of the hole $g$-factor can be traced back to the spatial distribution of the hole wave function in the dots.

The in-plane anisotropy of the hole $g$ factor can therefore

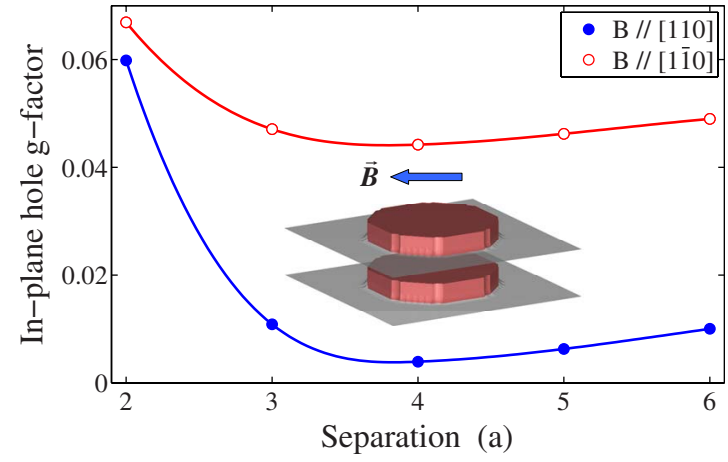

FIG. 5. (Color online) Hole $g$ factor in vertically coupled InAs/ GaAs self-assembled quantum dots calculated as a function of the separation between the dots for the magnetic field directed along the [110] (solid dots) and [1 $1 \overline{0}]$ (open dots) directions, respectively. Each disklike dots have a diameter of $15.3 \mathrm{~nm}$ and height of $2.8 \mathrm{~nm}$. The separation varies between $2 a$ and $6 a$ where $a$ is the lattice constant of GaAs.

be attributed to the contribution from the nonzero envelope orbital momenta carried by the hole states. For an electron state, the contribution from NEOM to its overall $g$ factor is negligible and hence it exhibits smaller anisotropy between Faraday and Voigt configuration ${ }^{14}$ and almost isotropic within the growth plane.

Besides for the single quantum dots, the hole states in coupled quantum dots have been revealed to exhibit very different $g$ factors from those of the electrons. ${ }^{15}$ Here we adopt the same model system as in our previous work ${ }^{16}$ to study the in-plane anisotropy of hole $g$ factors in coupled self-assembled quantum dots. Each of islands is disk-like and has a diameter of $15.3 \mathrm{~nm}$ and height of $2.8 \mathrm{~nm}$. The separation between the bottom of the upper dot and top of the lower dot varies between $1.1 \mathrm{~nm}$ and $3.4 \mathrm{~nm}$. Figure 5 plots the hole $g$ factors in Voigt configuration calculated as a function of the separation between the dots. It is seen that the in-plane hole $g$ factor exhibits larger anisotropy than those in the single quantum dots, though with similar amplitude. The major axis of the in-plane hole $g$ factor is found to be perfectly lying along the crystal $[1 \overline{1} 0]$ axis, which is different from that for the thin dots but, however, coinciding with the thick ones (see Fig. 1).

In the previous models for intermixed InGaAs quantum dots, random composition profile is assumed. In experiment, it has been found that the intermixing effect may lead to noticeably inhomogeneous composition distribution inside the quantum dots with an interesting pattern. ${ }^{17}$ Here, we adopt the same pattern for the inhomogeneous composition distribution. With the intermixing effect, the indium concentration decreases from $100 \%$ in the region close to the apex of the dot to about $40 \%$ in the bottom of the dot, resulting in a nominal composition of about $75.6 \%$. Each of the coupled truncated pyramidal dots has a base length of $19.8 \mathrm{~nm}$ and height $2.8 \mathrm{~nm}$. The separation varies between 2.3 and 4.5 $\mathrm{nm}$. First we find that the major axis of the in-plane hole $g$ factor is very close the [010] direction, very different from those obtained from previous models. Second, the amplitude of the in-plane hole $g$ factor is seen to be significantly larger. 
TABLE I. In-plane hole $g$ factors in coupled quantum dots.

\begin{tabular}{ccc}
\hline \hline Separation (a) & $g_{x}$ & $g_{y}$ \\
\hline 4 & 0.3997 & 0.5208 \\
6 & 0.3769 & 0.5125 \\
8 & 0.3516 & 0.5061 \\
\hline \hline
\end{tabular}

Table I lists the calculated in-plane hole $g$ factors against the separation between the dots (in the unit of GaAs lattice constant). Compared with those in pure InAs coupled dots, the in-plane hole $g$ factors in these intermixed structures are almost one order of magnitude larger in amplitude and much less in anisotropy. Such large in-plane hole $g$-factors with small anisotropy places a convincing scenario for efficient spin injection into self-assembled quantum dots. ${ }^{18}$ Besides the factors known in the previous studies, other effects such as spin-orbit interaction in the envelope functions of the carriers ${ }^{19}$ and Coulomb correlation ${ }^{20}$ may also play a role in determining the electron and hole $g$ factors in self-assembled quantum dots, which will be addressed in our further study.

In conclusion, we have studied the in-plane anisotropy of the hole $g$ factors in both single and coupled self-assembled quantum dots. Compared with isotropic electron spins within the growth plane, we have shown that the hole spins can be highly anisotropic. We have revealed that the in-plane anisotropy of the hole $g$ factors can be attributed to the contribution from the nonzero envelope orbital momenta carried by the hole states. We have found that the dependence of this contribution on the geometry of the dots can even reverse the sign of the in-plane anisotropy as the shape of the dots varies. In vertically coupled quantum dots with realistic intermixing composition profile, we have shown that the in-plane hole $g$-factors are comparable to the electrons and almost one order of magnitude larger than those in the single dots. Our result agrees qualitatively with the recent experiment.

This work is supported by the NSFC (Grants No. 10774025 and No. 60876087), the 973 project of MOST of China (Grant No. 2006CB921506), the STCSM (Grant No. 08jc14019).

\footnotetext{
*shengw@fudan.edu.cn

${ }^{1}$ B. van Wees, Nat. Phys. 3, 147 (2007).

${ }^{2}$ Semiconductor Spintronics and Quantum Computation, edited by D. D. Awschalom, D. Loss, and N. Samarth (Springer-Verlag, Heidelberg, 2002).

${ }^{3}$ W. Sheng and A. Babinski, Phys. Rev. B 75, 033316 (2007).

${ }^{4}$ W. Sheng, S. J. Xu, and P. Hawrylak, Phys. Rev. B 77, 241307(R) (2008).

${ }^{5}$ I. A. Yugova, A. Greilich, E. A. Zhukov, D. R. Yakovlev, M. Bayer, D. Reuter, and A. D. Wieck, Phys. Rev. B 75, 195325 (2007).

${ }^{6}$ M. Kroner, K. M. Weiss, B. Biedermann, S. Seidl, A. W. Holleitner, A. Badolato, P. M. Petroff, P. Öhberg, R. J. Warburton, and K. Karrai, Phys. Rev. B 78, 075429 (2008).

${ }^{7}$ S. A. Crooker, J. Brandt, C. Sandfort, A. Greilich, D. R. Yakovlev, D. Reuter, A. D. Wieck, and M. Bayer, Phys. Rev. Lett. 104, 036601 (2010).

${ }^{8}$ M. Graf and P. Vogl, Phys. Rev. B 51, 4940 (1995); T. B. Boykin, R. C. Bowen, and G. Klimeck, ibid. 63, 245314 (2001); T. B. Boykin and P. Vogl, ibid. 65, 035202 (2001); B. A. Foreman, ibid. 66, 165212 (2002).

${ }^{9}$ S. Raymond, S. Studenikin, A. Sachrajda, Z. Wasilewski, S. J.
}

Cheng, W. Sheng, P. Hawrylak, A. Babinski, M. Potemski, G. Ortner, and M. Bayer, Phys. Rev. Lett. 92, 187402 (2004).

${ }^{10}$ A. Babinski, M. Potemski, S. Raymond, J. Lapointe, and Z. R. Wasilewski, Phys. Rev. B 74, 155301 (2006).

${ }^{11}$ W. Sheng and J.-P. Leburton, Phys. Status Solidi B 237, 394 (2003).

${ }^{12}$ W. Sheng, Appl. Phys. Lett. 94, 123113 (2009).

${ }^{13}$ W. Sheng, Appl. Phys. Lett. 89, 173129 (2006).

${ }^{14}$ F. G. G. Hernandez, T. P. Mayer Alegre, and G. MedeirosRibeiro, Appl. Phys. Lett. 92, 132106 (2008).

${ }^{15}$ M. F. Doty, M. Scheibner, I. V. Ponomarev, E. A. Stinaff, A. S. Bracker, V. L. Korenev, T. L. Reinecke, and D. Gammon, Phys. Rev. Lett. 97, 197202 (2006).

${ }^{16}$ W. Sheng, Appl. Phys. Lett. 95, 113105 (2009).

${ }^{17}$ P. Offermans, P. M. Koenraad, J. H. Wolter, K. Pierz, M. Roy, and P. A. Maksym, Phys. Rev. B 72, 165332 (2005).

${ }^{18}$ W. Sheng and P. Hawrylak, Phys. Rev. B 73, 125331 (2006).

${ }^{19}$ M. Vachon, S. Raymond, A. Babinski, J. Lapointe, Z. Wasilewski, and M. Potemski, Phys. Rev. B 79, 165427 (2009).

${ }^{20}$ D. Y. Oberli, M. Byszewski, B. Chalupar, E. Pelucchi, A. Rudra, and E. Kapon, Phys. Rev. B 80, 165312 (2009). 